\title{
Immunoglobulin $M$ gene association with autoantibody reactivity and type 1 diabetes
}

\author{
Inês Rolim ${ }^{1,2}$ • Nádia Duarte ${ }^{1}$ - Gabriela Barata ${ }^{2,3}$ • João Costa ${ }^{1}$ • \\ Luís Gardete-Correia ${ }^{3}$ - José Boavida ${ }^{3}$ - Rui Duarte ${ }^{3}$. João Raposo ${ }^{3}$. \\ Zulmira Peerally $^{3} \cdot$ Manuela Catarino $^{2} \cdot$ Carlos Penha-Gonçalves ${ }^{1,3}$
}

Received: 6 April 2017 / Accepted: 4 May 2017 / Published online: 22 May 2017

(C) The Author(s) 2017. This article is an open access publication

\begin{abstract}
Several lines of evidence show that autoimmune responses evolving in type 1 diabetes (T1D) patients include the generation of multi-reactive autoantibody (AutoAb) repertoires, but their role in T1D pathogenesis remains elusive. We tested the hypothesis that variants at the immunoglobulin heavy chain (IGH) locus are genetic determinants of AutoAbs against pancreatic antigens and contribute to T1D susceptibility. With this aim, two independent study designs were used: a case-control study and a family-based cohort comprising a total of 240 T1D patients, 172 first-degree relatives (mother and/or father), and 130 unrelated healthy controls living in Portugal. We found that three SNPs in the IGH locus show suggestive association with T1D with the highest nominal association at rs1950942 (in the IGHM-IGHJ gene region) in both the case-control study $(P=9.35 \mathrm{E}-03)$ and the family-based cohort $(P=3.08 \mathrm{E}-03)$. These SNPs were also associated with IgG AutoAbs against pancreatic antigens and with AutoAb multi-reactivity in T1D patients. Notably, we found that the SNP with the highest association with T1D susceptibility and IgG autoantibody reactivity (rs1950942) was also associated with anti-GAD IgM reactivity in T1D patients $(P=5.98 \mathrm{E}-03)$ and in non-affected parents
\end{abstract}

Electronic supplementary material The online version of this article (doi:10.1007/s00251-017-0999-1) contains supplementary material, which is available to authorized users.

Carlos Penha-Gonçalves

cpenha@igc.gulbenkian.pt

1 Instituto Gulbenkian de Ciência, Apartado 14, P-2781-901 Oeiras, Portugal

2 Faculdade de Farmácia, Universidade de Lisboa, Lisbon, Portugal

3 Portuguese Diabetes Association, Education and Research Center, Lisbon, Portugal
( $P=4.17 \mathrm{E}-03)$. This finding implies that IGH association with autoreactive $\operatorname{IgM}$ is detectable irrespective of disease status.

These results suggest that genetic variants at the IgM gene region of the IGH locus contribute to antibody autoreactivity and are associated with T1D. We propose that the control of autoantibody generation by $I G H$ polymorphisms is a component of the complex architecture of T1D genetic susceptibility.

Keywords IgH locus · Type 1 diabetes - Autoantibodies . Anti-GAD $\cdot \operatorname{IgM} \cdot$ Genetic association

\section{Introduction}

Autoantibodies (AutoAbs) have proved to be pathogenic in several autoimmune diseases, but their role in type 1 diabetes (T1D) remains elusive (Notkins and Åke, 2001; Elliott et al. 2012). Autoimmune reactions against insulin-producing beta cells, in the pancreatic islets of Langerhans, are critical T1D pathogenic events where $T$ cell-mediated activity is perceived to play a key role in the effector phase of beta cell destruction (Bach 1994; Roep and Peakman 2011). Nevertheless, B cells and the antibodies they secrete are not negligible. B cells were shown to contribute to beta cell autoimmunity in the NOD mouse model by presenting islet autoantigens to $\mathrm{T}$ cells or by secreting proinflammatory cytokines (Chan and Shlomchik 1998; Chan et al. 1999; Harris et al. 2000; Wong et al. 2004). In addition, B cell depletion in clinical trials showed beneficial effects in preserving beta cell function (Pescovitz et al. 2009). On the other hand, AutoAbs in T1D represent a clear manifestation that B cell tolerance has been disrupted (Ziegler et al. 1999; Kimpimäki et al. 2002; LaGasse et al. 2002). AutoAbs against islet antigens are detectable at very early pathogenesis stages and are instrumental indicators 
of preclinical diabetes progression (Yu et al. 2000). Anti-islet antibodies can enhance the expansion of islet-reactive $\mathrm{CD} 4^{+} \mathrm{T}$ cells and contribute to disease progression in a T1D transgenic mouse model (Harbers et al. 2007). Furthermore, anti-islet AutoAbs were shown to trigger murine autoimmune diabetes in the presence of an increased frequency of islet-reactive CD4 T cells (Silva et al. 2011).

AutoAb repertoires in T1D patients often display specificities typical of other autoimmunity diseases. Reportedly, T1D patients show high prevalence of concomitant autoimmune thyroid disease (15 to 30\%) (Perros et al. 1995; Umpierrez et al. 2003), celiac disease (4-9\%) (Bao et al. 1999; Carlsson et al. 1999; Aktay et al. 2001; Barera et al. 2002; Barker et al. 2005), or Addison's disease (0.5\%) (Barker et al. 2005). A large study demonstrated in T1D patients that occurrence of AutoAbs against antigens a priori not associated with diabetes is in part genetically controlled by non-HLA T1D susceptibility loci (Plagnol et al. 2011). These results reinforce the view that autoimmune responses evolving in T1D patients include the generation of multi-reactive AutoAb repertoires.

At large extent, the AutoAb research in T1D focuses on total IgG antibodies against anti-islet antigens, which result from adaptive immune responses. Nevertheless, high prevalence of IgG1 and IgG3 AutoAbs were associated with rapid progression to T1D (Hoppu et al. 2004a), indicating that different Ig isotypes may have distinct roles in disease. Although the IgM isotype is represented among the AutoAbs implicated in T1D, its association with disease pathogenesis is unclear (Dean et al. 1986; Hawa et al. 2000). It has been reported that the human fetus generates natural antibodies that recognize a uniform set of autoantigens, some of which are associated with autoimmune diseases (Merbl et al. 2007). Natural antibodies are produced in the absence of exogenous stimulation and are germline-encoded, low-affinity, polyreactive antibodies, mainly of the IgM isotype (Coutinho et al. 1995). This raises the possibility that faltering generation of natural and otherwise benign autoimmunity could result in autoimmune disease (Merbl et al. 2007). We have reported that the IgM repertoire of pre-insulitic NOD mice displays increased levels of B1 cell-derived antiislet antibodies that are able to bind beta cells ex vivo and are generated irrespective of the NOD major histocompatibility haplotype (H-2) (Côrte-Real et al. 2012). Furthermore, it has been demonstrated that the murine repertoire of immunoglobulin heavy chain $(\mathrm{IgH})$ gene rearrangements is dependent on the $I g H$ haplotype (Viale et al. 1992) and is abnormal in the NOD mouse (Andersson et al. 1994).

We have noted that genome-wide SNP genotyping platforms show poor coverage of common frequency polymorphisms in the IGH locus (Supp. Table S1). This prompted us to investigate the role of the $I G H$ locus in the genetic determination of AutoAb repertoires and in T1D susceptibility. Here, we tested the genetic association of $I G H$ SNPs to T1D susceptibility and to antibody autoreactivity in two cohorts of Portuguese patients.

\section{Materials and methods}

\section{Subjects and clinical criteria}

This investigation was conducted under the ethical permission obtained for the study entitled "Estudo da base genética da imunopatologia associada à Diabetes Tipo 1 na população portuguesa" granted by the Ethics Committee of the Associação Protectora dos Diabéticos de Portugal (APDP) in Lisbon. Written, informed consent was obtained from the participants or parents of each child. All investigations have been conducted according to the principles expressed in the Declaration of Helsinki. Patients were selected among attendance to the APDP, and sample collection was carried out from April 2007 to August 2009 comprising a total of 240 T1D patients, 167 first-degree relatives (mother and/or father), and 130 unrelated healthy controls living in Portugal. Ninety-seven percent of the patients were Caucasian. Type 1 diabetes diagnosis met the criteria established by the American Diabetes Association. In the family-based collection, the inclusion criteria selected patients with less than 5 years of disease duration.

\section{Autoantibody analysis}

A total of 227 patients and 146 non-affected parents were analyzed for IgM anti-glutamic acid decarboxylase antibodies (IgM anti-GAD) by indirect enzyme-linked immunosorbent assay (ELISA). Briefly, flat-bottomed 96-well ELISA plates were coated overnight at $4{ }^{\circ} \mathrm{C}$, with $1 \mu \mathrm{g} / \mathrm{mL}$ glutamic acid decarboxylase (GAD65/67 C-terminal) peptide (ENZO Life Sciences Inc., Farmingdale, NY, USA) in coating buffer (0.05 M K2PO4). The plates were washed in PBS-Tween, blocked with PBS-BSA $3 \%$, and incubated at $37{ }^{\circ} \mathrm{C}$ for $120 \mathrm{~min}$. After washing, four serial dilutions of sera $(1: 25$, $1: 50,1: 100,1: 200$ ) were incubated for $180 \mathrm{~min}$ at $37^{\circ} \mathrm{C}$ and washed. Bound IgM was detected by incubation with $6 \mu \mathrm{g} /$ $\mathrm{mL}$ biotin mouse anti-human IgM antibody (BD Biosciences, Franklin Lakes, NJ, USA) in PBS-Gelatin 1\%-Tween $0.075 \%$ (1:4000), $4{ }^{\circ} \mathrm{C}$ overnight, followed by incubation with streptavidin AKP (1:1000) (Biolegend, San Diego, CA, USA), $37^{\circ} \mathrm{C}$ for $2 \mathrm{~h}$, and revealed with the $1 \mu \mathrm{g} / \mathrm{mL}$ pNPP substrate (Sigma-Aldrich, St. Louis, MO, USA). The absorbance at $405 \mathrm{~nm}$ was determined using a micro-ELISA plate reader and results were expressed in arbitrary units (AU) calculated as follows: first, sample absorbance was normalized to the absorbance of the serum of a diagnosed T1D patient (positive reference) that was run in all Elisa plates. Second, we corrected this value for inter-plate variation using one sample of a randomly chosen healthy individual (negative reference) that was also run in all the plates. Correction for the variation coefficient of the negative reference was performed according to the following formula: Corrected value $=$ normalized 
value $\times(1-\mathrm{CV})$, where $\mathrm{CV}$ is the coefficient of variation of the negative reference. Patients and relatives were also analyzed for IgG AutoAb seropositivity using commercial standardized clinical laboratory tests for the following antigens: protein tyrosine phosphatase (PTP)-like protein (IA-2 ELISA Version 2 kit, RSR, Cardiff, UK): specificity $99 \%$ and sensitivity 63\%; glutamic acid decarboxilase (GAD65 Ab ELISA kit, RSR): specificity $98 \%$ and sensitivity $92 \%$; and islet cell autoantigens (ICA, Indirect Imunofluorescence, Menarini, Florence, Italy).

\section{IGH SNP genotyping}

Genomic DNA was extracted from whole blood using the Chemagen Magnetic Bead Technology (Chemagic MSM I, Baesweiler, Germany). DNA preparations were quantified using the PicoGreen method (Invitrogen/Life Technologies, Paisley, UK) according to supplier instructions. SNP selection criteria took into account allelic frequency in the Caucasian populations, available sequencing confirmation, and tagging of LD blocks (http://www.hapmap.org). The SNP genotyping method used the Mass Array system to design multiplex reactions for PCR, iPlex primer extension (Sequenom, San Diego, CA, USA) and the MALDI-TOF-based Mass Array platform (Sequenom). A total of 239 patients, 169 nonaffected parents, and 130 unrelated healthy controls were genotyped for 15 SNPs mapping in 4 regions in immunoglobulin heavy chain locus: 4 SNPs in the $\operatorname{IgHG}, 4$ in $\operatorname{IgHD}, 5$ in $\operatorname{IgHM}$, and 2 in IgHV regions. Genotyping quality control selected 9 SNPs that yield correct genotyping data according to HapMap control samples and passed the Hardy-Weinberg equilibrium test $(P>0.05)$ with a call rate above $80 \%$.

\section{Genetic analysis}

Analysis of T1D association was performed in two independent datasets. At nominal 0.05 significance level, the casecontrol dataset has $85 \%$ power to detect allelic association effects assuming OR 1.25, 30\% allele frequency, and complete LD with trait while the family-based dataset has $74 \%$ power to detect allelic transmission disequilibrium assuming OR 2.5, 30\% allele frequency, and complete LD with trait. T1D association in the case-control dataset was performed under different genetic models using the PLINK software, and results of the best fitting model are presented (Purcell et al. 2007). Genetic association in the family-based cohort was analyzed with transmission disequilibrium tests (TDT) using the PLINK TDT software. Linkage disequilibrium (LD) analysis was performed using the correlation coefficient $\left(r^{2}\right)$ to estimate pair-wise LD. The LD map was plotted using the Haploview software to identify LD blocks. Genetic association with IgG AutoAbs was tested using PLINK TDT. Association analysis of anti-GAD IgM titer was performed by quantitative trait loci (QTL) methods implemented in PLINK. Nominal results under the conventional $P$ value $=0.05$ were considered suggestive evidence for association.

\section{Results}

\section{Study design}

We sought to analyze the association of SNPs at the $I G H$ locus with T1D and in two patient cohorts. Despite the paucity of markers with common alleles in this region, we identified 9 SNPs spanning $400 \mathrm{~kb}$ in the $I G H$ locus that passed genotyping quality control criteria (Fig. 1a). In the case-control collection, we compared genotype frequencies of $9 \mathrm{IgH}$ SNPs in 137 patients with 130 healthy controls. Using the family-based collection, we performed transmission disequilibrium tests (TDT) in 102 recently diagnosed T1D patients and 169 non-affected parents. $I g H$ SNPs showing suggestive association with T1D in either collection were used to test association with autoreactive antibodies. IgG AutoAb positivity association analysis used TDT in the family-based cohort, while quantitative trait analysis (QTL) of IgM AutoAb titer used either T1D cases of both cohorts and non-affected parents (Table 1). No correlation was found between AutoAb levels at enrollment and age at diagnosis or time since diagnosis (data not shown).

\section{IGH locus association with T1D}

Case-control genotype association analysis identified four IGH SNPs (rs17113415, rs2180790, rs1956596, and rs1950942) showing suggestive association with T1D $(P<0.05)$, which best fitted the recessive model. Three associated SNPs mapped in the IGHM gene segment coding for the immunoglobulin $\mathrm{M}$ isotype (Fig. 1b, Table 2). Accordingly, transmission disequilibrium analysis in the parent-affected child cohort detected that the same three SNPs in the IGHM gene (rs2180790, rs1956596, and rs1950942) were associated with T1D (Fig. 1b, Table 3). In these two independent cohorts, rs1950942 showed the highest association $(P=9.35 \mathrm{E}-03$ and $P=3.08 \mathrm{E}-03$, respectively), and these concordant results denote that the identified associations are not attributable to population stratification effects. Linkage disequilibrium (LD) analysis revealed a region of strong LD covering $15 \mathrm{~kb}$ of the IGHD and IGHM regions that encompassed the four SNPs associated with T1D, suggesting a single association signal (Fig. 1b). We noted that an additional SNP (rs1808152) mapping $57 \mathrm{~kb}$ upstream from this LD block (in the region of $I G H$ diversity segments) was over-transmitted to T1D patients (Table 3 ) and could represent a risk factor in T1D. The coherent results in the two cohorts corroborate that genetic polymorphisms closely mapping in the $I G H M$ region show suggestive association with T1D. 
Fig. 1 Genetic association of immunoglobulin heavy chain $(I G H)$ locus to type 1 diabetes (T1D). a Relative physical distances of nine single nucleotide polymorphisms (SNP) across $430 \mathrm{~kb}$ in $I G H$ locus, in chromosome 14. SNP assignment to $I G H$ gene segments is shown (see Table 2 for individual SNPdefined positions). b Nominal $P$ values of association tests in two cohorts of T1D patients. Case control $(C C)$ genotypic analysis under the recessive model was performed in 137 patients and 130 controls and is represented by the inverted shaded triangles.

Transmission disequilibrium tests (TDT) were performed in $102 \mathrm{pa}-$ tients and 169 first-degree relatives and are represented by the open circles. Dashed line indicates the threshold of suggestive association $(P=0.05)$. Linkage disequilibrium $(L D)$ analysis is depicted as a diagram generated by the Haploview software package and values of pair-wise $r$ squared are represented. The dark shaded area represents a LD block encompassing five analyzed SNPs in the $I G H D-M$ region a

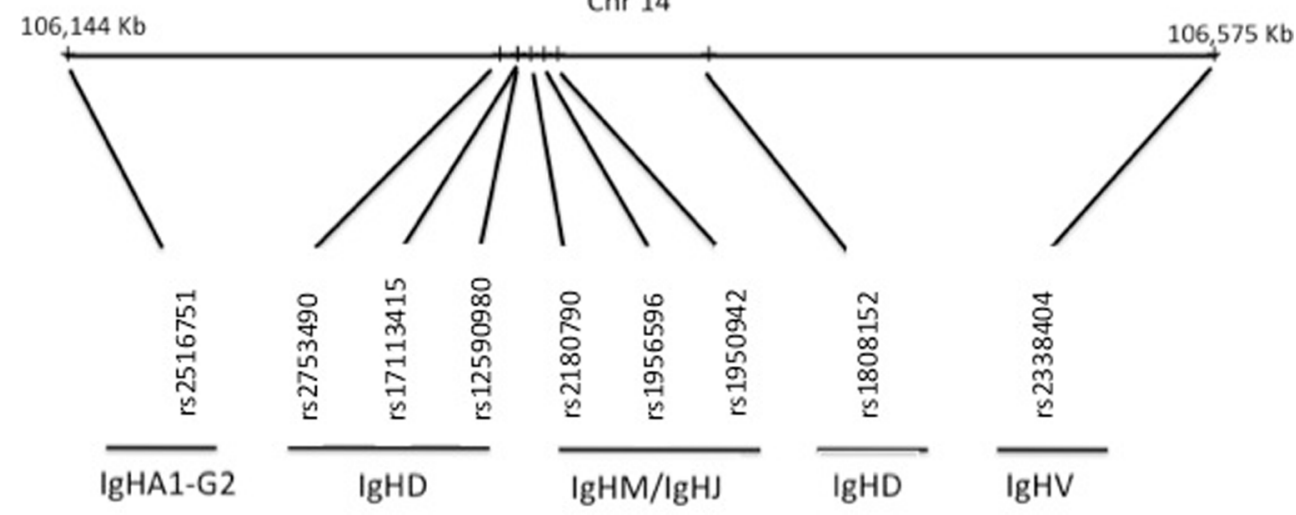

b
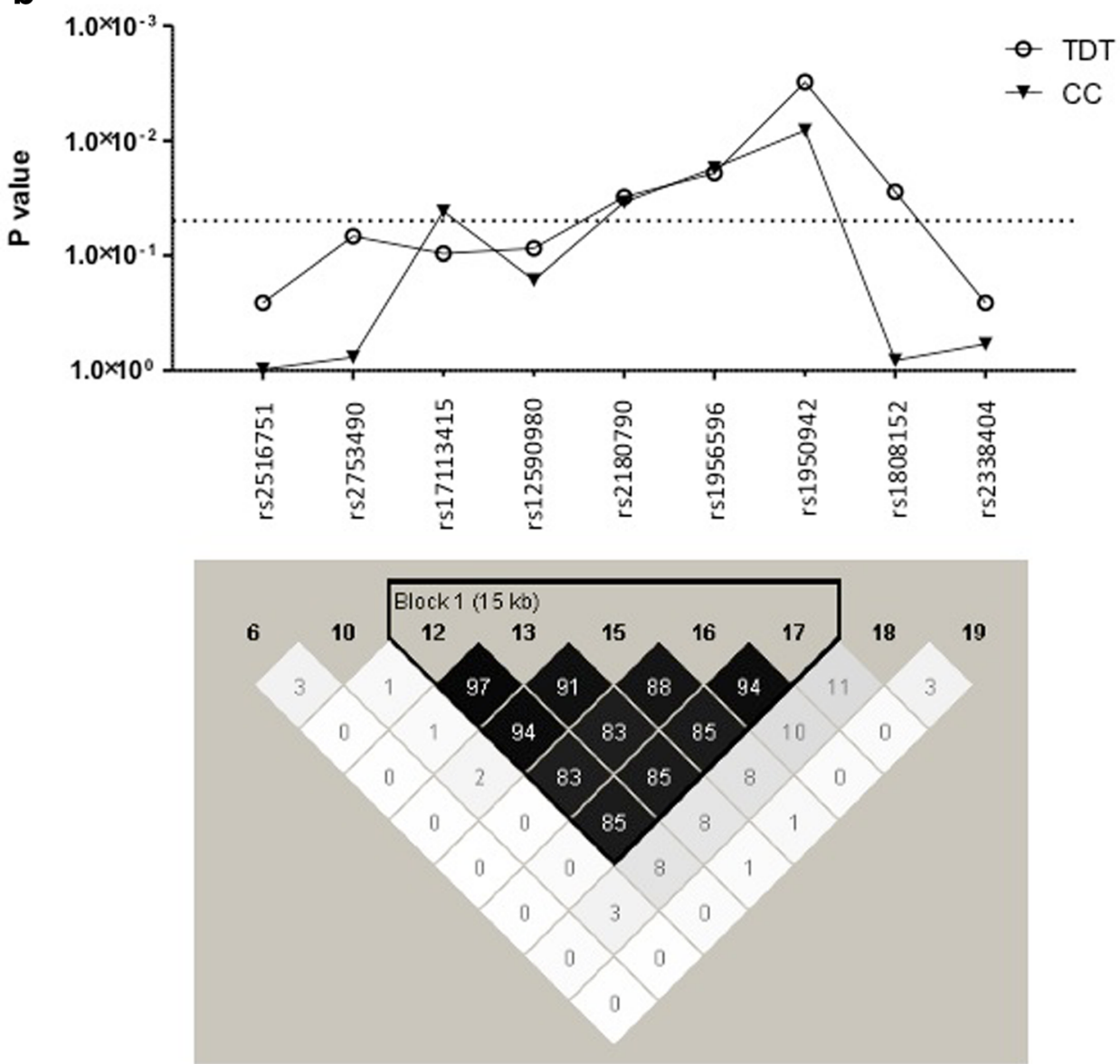

\section{IGH region and autoantibody repertoires}

Next, we tested whether the IGHM SNPs associated with T1D were also predisposing to antibody positivity in T1D patients. We analyzed 76 patients and 126 unaffected parents of the family-based cohort for three $\operatorname{IgG}$ AutoAbs associated with T1D, namely anti-GAD, anti-ICA, and anti-IA-2. The prevalence of these AutoAbs in our patient collection varied from 50 to $75 \%$, while was considerably lower in their unaffected relatives $(<11 \%)$ (Fig. 2a). AutoAb positivity against each of the three pancreatic-islet antigens was suggestively associated with three SNPs that were also associated with T1D (rs1956596, rs1950942, and rs1808152) (Table 4 and Supp. Tables S2 and S3) suggesting that presence of AutoAbs for multiple antigens could be partially controlled by the $\operatorname{IgH}$ locus.

To determine whether the IGHM region was controlling repertoire multi-autoreactivity, we classified patients as mono-autoreactive (if positive for any one Auto $\mathrm{Ab}$ ) or as multi-autoreactive (if positive for two or three AutoAbs). 
Table 1 Study plan and sample size

\begin{tabular}{|c|c|c|c|c|c|}
\hline Phenotype & Type 1 diabetes & & IgG anti-GAD & $\operatorname{IgG} A A b s^{e}$ & IgM anti-GAD \\
\hline Genetic test & Genot. Assoc. ${ }^{\mathrm{c}}$ & $\mathrm{TDT}^{\mathrm{d}}$ & $\mathrm{TDT}^{\mathrm{d}}$ & $\mathrm{TDT}^{\mathrm{d}}$ & $\mathrm{QTL}^{\mathrm{f}}$ \\
\hline Sample size & 137 cases 130 controls & 102 cases 169 relatives & 57 cases 126 relatives & 49 cases 126 relatives & 227 cases 146 relatives \\
\hline Age in cases (years) ${ }^{\mathrm{a}}$ & $31.7 / 29$ & $19.1 / 17.4$ & $18.0 / 17.0$ & $16.5 / 16$ & $27.3 / 25.0$ \\
\hline Age-at-diagnosis (years) ${ }^{\mathrm{b}}$ & $18.4 / 18.0(2-51)$ & $17.4 / 16.8(2-28)$ & $15.3 / 15.8(2-30)$ & $14.4 / 15.0(2-23)$ & $17.4 / 16.4(2-47)$ \\
\hline Disease duration (years) ${ }^{\mathrm{a}}$ & $13.3 / 12.0$ & $1.7 / 1.0$ & $2.6 / 1.0$ & $1.8 / 1.0$ & $9.9 / 7.0$ \\
\hline Sex: male patients $(\%)$ & 56 & 63 & 54 & 59 & 56 \\
\hline
\end{tabular}

${ }^{\text {a }}$ Mean/median

${ }^{\mathrm{b}}$ Mean/median (age range)

${ }^{\mathrm{c}}$ Genotype association analysis

$\mathrm{d}$ Transmission disequilibrium test

${ }^{\mathrm{e}}$ Multiple autoantibodies

${ }^{\mathrm{f}}$ Quantitative trait locus analysis

This showed that approximately $77 \%$ of the T1D patients were multi-autoreactive (Fig. 2b). Using multi-autoreactivity as affected status, we found that rs1950942 in the IGHM region was associated with antibody multi-autoreactivity in T1D patients, as ascertained by TDT (Table 5). Strikingly, this SNP also showed the strongest association with T1D (Fig. 1, Tables 2 and 3), suggesting that its contribution to T1D relates to the generation of multi-autoreactive antibody repertoires.

The location of rs 1950942 in the IGHM region indicated that this region could control the IgM autoantibody repertoire in T1D patients. Thus, we ascertained serum IgM autoreactivity by measuring anti-GAD reactivity in T1D patients and nonaffected parents, with a GAD peptide-based ELISA assay. This anti-GAD ELISA detects low-affinity IgM antibodies, as prior serum incubation with soluble GAD did not impede the detection of anti-GAD reactivity (data not shown). We found that T1D patients had significantly higher levels of anti-GAD reactivity as compared to non-affected parents (Fig. 3). Performing QTL analysis under the additive model in 227 T1D patients, we found that rs1950942 was controlling the levels of anti-GAD IgM reactivity (Table 6). The same SNP was found to control anti-GAD IgM reactivity in non-affected parents (Table 7), suggesting that the $I G H$ genetic control of anti-GAD IgM production was not conditioned by the T1D status. This genetic effect is illustrated by the rs 1950942 genotype-stratified analysis of anti-GAD IgM levels in patients and relatives (Fig. 4). Together, these data implicate polymorphisms in the $I g M$ region of the $I G H$ locus in the generation of IgM and IgG AutoAbs in T1D patients and cohesively suggest the involvement of the IGHM locus in disease susceptibility.

Table $2 I G H$ SNPs and type 1 diabetes association with the case-control cohort

\begin{tabular}{|c|c|c|c|c|c|c|c|c|c|}
\hline MARKER & Chr14 (bp) $)^{\mathrm{a}}$ & Gene region & Call rate ${ }^{\mathrm{b}}$ & Minor allele & $\mathrm{MAF}^{\mathrm{c}}$ & Cases $^{\mathrm{d}}$ & Controls ${ }^{\mathrm{d}}$ & OR $(95 \% \mathrm{CI})^{\mathrm{e}}$ & $P$ \\
\hline $\operatorname{rs} 2516751$ & $106,143,806$ & $I G H A 1 / I G H G 2$ & 0.87 & G & 0.48 & $30 / 106$ & $21 / 75$ & $1.01(0.53-1.90)$ & $9.73 \mathrm{E}-01$ \\
\hline rs2753490 & $106,306,394$ & $I G H D$ & 0.96 & $\mathrm{~T}$ & 0.25 & $8 / 122$ & $9 / 118$ & $0.860 .32-2.30)$ & $7.64 \mathrm{E}-01$ \\
\hline rs17113415 & $106,312,884$ & $I G H D$ & 0.95 & $\mathrm{~T}$ & 0.44 & $22 / 110$ & $33 / 88$ & $0.53(0.29-0.98)$ & $4.11 \mathrm{E}-02$ \\
\hline rs 12590980 & $106,313,201$ & $I G H D$ & 0.92 & A & 0.43 & $22 / 114$ & $26 / 86$ & $0.64(0.34-1.20)$ & $1.64 \mathrm{E}-01$ \\
\hline rs2180790 & $106,317,994$ & IGHM downst & 0.96 & $\mathrm{~T}$ & 0.46 & $22 / 109$ & $35 / 91$ & $0.52(0.29-0.96)$ & $3.36 \mathrm{E}-02$ \\
\hline rs1956596 & $106,322,972$ & $I G H M$ upst & 0.96 & G & 0.48 & $24 / 105$ & $40 / 87$ & $0.50(0.28-0.89)$ & $1.83 \mathrm{E}-02$ \\
\hline rs1950942 & $106,328,066$ & IGHM/ IGHJ6 & 0.92 & G & 0.43 & $15 / 103$ & $33 / 93$ & $0.41(0.21-0.80)$ & $9.35 \mathrm{E}-03$ \\
\hline rs1808152 & $106,384,914$ & $I G H D 1-1$ & 0.88 & $\mathrm{C}$ & 0.34 & $12 / 104$ & $14 / 110$ & $0.91(0.40-2.05)$ & $8.14 \mathrm{E}-01$ \\
\hline rs2338404 & $106,575,151$ & $I G H V$ & 0.91 & $\mathrm{~A}$ & 0.40 & $19 / 115$ & $19 / 95$ & $0.83(0.41-1.65)$ & $5.88 \mathrm{E}-01$ \\
\hline
\end{tabular}

${ }^{\text {a }}$ Positioning in chrom14 (EnsemblGRCh37)

${ }^{\mathrm{b}}$ All individuals in the two cohorts

${ }^{\mathrm{c}}$ Minor allele frequency

${ }^{\mathrm{d}}$ Minor allele homozygous genotypes/alternative genotypes

${ }^{\mathrm{e}}$ Odds ratio and $95 \%$ confidence interval 
Table 3 Type 1 diabetes: transmission disequilibrium test for $I G H$ SNPs

\begin{tabular}{llllllll}
\hline Marker & MFA $^{\mathrm{a}}$ & Trans $^{\mathrm{b}}$ & Untrans $^{\mathrm{c}}$ & OR $^{\mathrm{d}}$ & L95 $^{\mathrm{e}}$ & U95 $^{\mathrm{f}}$ & $P$ \\
\hline rs2516751 & A & 11 & 17 & 0.64 & 0.30 & 1.38 & $2.57 \mathrm{E}-01$ \\
rs2753490 & $\mathrm{T}$ & 10 & 20 & 0.50 & 0.23 & 1.06 & $6.79 \mathrm{E}-02$ \\
$\mathrm{rs} 17113415$ & $\mathrm{~T}$ & 13 & 23 & 0.56 & 0.28 & 1.11 & $9.56 \mathrm{E}-02$ \\
$\mathrm{rs} 12590980$ & $\mathrm{~A}$ & 12 & 22 & 0.54 & 0.27 & 1.10 & $8.64 \mathrm{E}-02$ \\
$\mathrm{rs} 2180790$ & $\mathrm{~T}$ & 14 & 28 & 0.50 & 0.26 & 0.94 & $3.08 \mathrm{E}-02$ \\
rs1956596 & $\mathrm{G}$ & 13 & 28 & 0.46 & 0.24 & 0.89 & $1.92 \mathrm{E}-02$ \\
rs1950942 & $\mathrm{G}$ & 8 & 25 & 0.32 & 0.14 & 0.71 & $3.08 \mathrm{E}-03$ \\
rs1808152 & $\mathrm{C}$ & 18 & 7 & 2.57 & 1.07 & 6.15 & $2.78 \mathrm{E}-02$ \\
rs2338404 & A & 17 & 11 & 1.54 & 0.72 & 3.10 & $2.57 \mathrm{E}-01$ \\
\hline
\end{tabular}

${ }^{\mathrm{a}}$ Minor frequency allele

${ }^{\mathrm{b}}$ Minor allele transmission count

${ }^{\mathrm{c}}$ Minor allele transmission count

${ }^{\mathrm{d}}$ Odds ratio

${ }^{\mathrm{e}}$ Lower limit of $95 \%$ confidence interval

${ }^{\mathrm{f}}$ Upper limit of $95 \%$ confidence interval

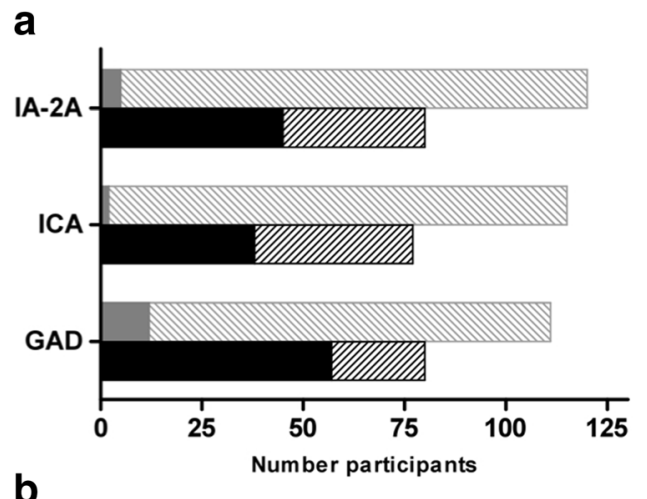

b

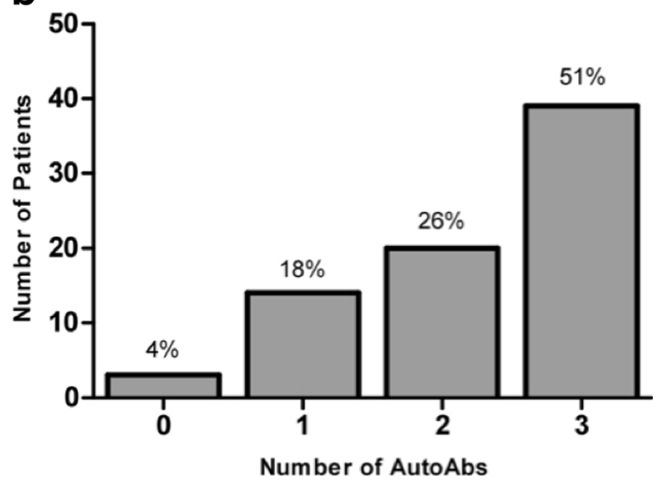

Fig. 2 Autoantibodies positivity. a Number of T1D patients of the family-based cohort (represented in black) or their non-affected parents (represented in gray) displaying IgG positivity (filled area) or negativity (striped area) for the indicated antigens. b Number of T1D patients of the family-based cohort showing positivity for the indicated number of antibody specificities against the following antigens: glutamic acid decarboxylase $(G A D)$, islet cell autoantigen $(I C A)$, and protein tyrosine phosphatase $(P T P)$-like protein (IA-2). Relative frequency of each group is shown as percent of the number of patients analyzed
Table 4 Anti-GAD IgG: transmission disequilibrium test for T1Dassociated SNPs in the $I G H$ locus

\begin{tabular}{llllllll}
\hline MARKER & MFA $^{\mathrm{a}}$ & Trans $^{\mathrm{b}}$ & Untrans $^{\mathrm{c}}$ & $\mathrm{OR}^{\mathrm{d}}$ & $\mathrm{L}^{\mathrm{e}}$ & $\mathrm{U}^{\mathrm{e}} 5^{\mathrm{f}}$ & \multicolumn{1}{l}{$P$} \\
\hline $\mathrm{rs} 2180790$ & $\mathrm{~T}$ & 7 & 13 & 0.54 & 0.21 & 1.35 & $1.80 \mathrm{E}-01$ \\
$\mathrm{rs} 1956596$ & $\mathrm{G}$ & 4 & 12 & 0.33 & 0.11 & 1.034 & $4.55 \mathrm{E}-02$ \\
rs1950942 & $\mathrm{G}$ & 3 & 12 & 0.25 & 0.07 & 0.88 & $2.01 \mathrm{E}-02$ \\
$\mathrm{rs} 1808152$ & $\mathrm{C}$ & 9 & 2 & 4.5 & 0.97 & 20.8 & $3.48 \mathrm{E}-02$ \\
\hline
\end{tabular}

${ }^{\mathrm{a}}$ Minor frequency allele

${ }^{\mathrm{b}}$ Minor allele transmission count

${ }^{\mathrm{c}}$ Minor allele non-transmission count

${ }^{\mathrm{d}}$ Odds ratio

${ }^{\mathrm{e}}$ Lower limit of $95 \%$ confidence interval

${ }^{\mathrm{f}}$ Upper limit of $95 \%$ confidence interval

\section{Discussion}

We report that polymorphisms in the $I G H M$ gene region show suggestive association with T1D development and with AutoAb repertoires. Nevertheless, studies on genetic association of AutoAbs in T1D that used data from genome-wide SNP scans did not identify the $I G H$ locus (Plagnol et al. 2011). We reason that current human genome variation databases incorporate an unusual scarcity of consensus SNPs at the $I G H$ locus. Consequently, the $I G H$ locus and in particular the $I G H$ constant region, determining the Ig isotype class, are poorly represented in the SNP arrays commonly used in GWAs studies (Supp. Table S1). We have hand-picked 15 SNPs spanning approximately $400 \mathrm{~kb}$ from the IGHA gene segment to the $\mathrm{V}$ region in the $I G H$ locus, but only 9 SNPs were successfully genotyped. This possibly reflects expected difficulties in obtaining SNPs with high allele call rate at this locus, which undergoes somatic genetic rearrangements represented in peripheral blood B cells.

The analysis of two independent T1D Portuguese cohorts replicated the association of T1D development with three

Table 5 Multi-autoreactive IgG: transmission disequilibrium test for T1D-associated SNPs in the $I G H$ locus

\begin{tabular}{llllllll}
\hline MARKER & MFA $^{\mathrm{a}}$ & Trans $^{\mathrm{b}}$ & Untrans $^{\mathrm{c}}$ & OR $^{\mathrm{d}}$ & L95 $^{\mathrm{e}}$ & U95 $^{\mathrm{f}}$ & $P$ \\
\hline rs2180790 & $\mathrm{T}$ & 10 & 15 & 0.66 & 0.30 & 1.48 & $3.17 \mathrm{E}-01$ \\
$\mathrm{rs} 1956596$ & $\mathrm{G}$ & 7 & 16 & 0.43 & 0.18 & 1.06 & $6.06 \mathrm{E}-02$ \\
$\mathrm{rs} 1950942$ & $\mathrm{G}$ & 4 & 14 & 0.28 & 0.09 & 0.87 & $1.84 \mathrm{E}-02$ \\
$\mathrm{rs} 1808152$ & $\mathrm{C}$ & 10 & 3 & 3.33 & 0.91 & 12.1 & $5.22 \mathrm{E}-02$
\end{tabular}

${ }^{a}$ Minor frequency allele

${ }^{\mathrm{b}}$ Minor allele transmission count

${ }^{\mathrm{c}}$ Minor allele non-transmission count

${ }^{\mathrm{d}}$ Odds ratio

${ }^{\mathrm{e}}$ Lower limit of $95 \%$ confidence interval

${ }^{\mathrm{f}}$ Upper limit of $95 \%$ confidence interval 


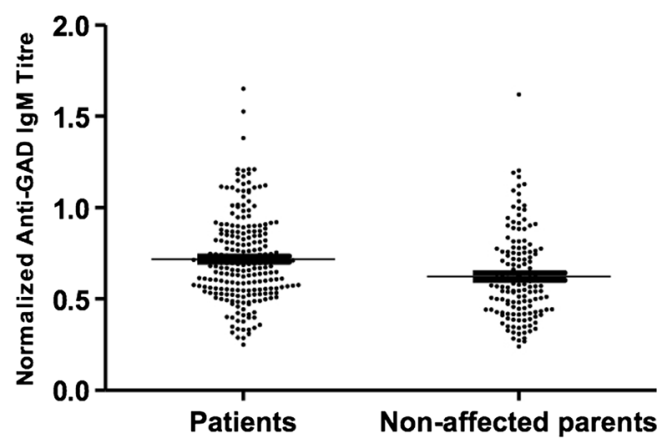

Fig. 3 Anti-GAD IgM reactivity. Results from 227 T1D patients and 146 non-affected parents are represented as arbitrary units after normalization, as described in the "Materials and methods" section. $P<0.0001$, Wilcoxon signed-rank test

SNPs mapping in the IGHM gene region (coding for the IgM isotype). Linkage disequilibrium analysis revealed that these three SNPs were encompassed within a 15-kb region of strong LD block together with one SNP in the IGHD gene ( $\operatorname{IgD}$ isotype) that showed marginal T1D association in the casecontrol cohort. Although these cohorts have relatively small sizes, these cohesive findings are suggestive of genetic association of the IGHM region with T1D. Nevertheless, the reported results warrant studies in larger cohorts to test replication of such T1D association signals in other populations. It is worth mentioning that despite very low IGH coverage, one genome-wide scan found that rs 1981496 (mapping within the $I G H V$ region and $250 \mathrm{~kb}$ upstream of LD block here reported) was associated with T1D at a significance level of $P=9.95 \mathrm{E}$ -05 (Burton et al. 2007). Although imputation analysis on GWAS data was not feasible due to scarcity of SNPs in this region, this observation corroborates the possibility that the IGH locus encloses association signals to T1D susceptibility.

Interestingly, the T1D association results highlighted rs 1950942 in a region close to an $I G H$ somatic recombination site at the boundary of the $I G H M$ and $I G H \mathrm{~J}$ regions. As $I G H$ recombination sites are major determinants of antibody specificity, our results point to a link of $I G H$ variants with the generation of AutoAb repertories in T1D. Genetic determination of self-reactivity should be detectable in primary repertoires, represented by the IgM low-affinity antibodies that do not result

Table 6 Anti-GAD IgM reactivity QTL analysis in T1D patients

\begin{tabular}{lllll}
\hline MARKER & NMISS $^{\mathrm{a}}$ & BETA $^{\mathrm{b}}$ & $r^{2 \mathrm{c}}$ & $P^{\mathrm{d}}$ \\
\hline rs2180790 & 216 & -0.021 & 0.010 & $1.39 \mathrm{E}-01$ \\
rs1956596 & 213 & -0.027 & 0.016 & $6.56 \mathrm{E}-02$ \\
rs1950942 & 200 & -0.042 & 0.037 & $5.98 \mathrm{E}-03$ \\
rs1808152 & 191 & -0.004 & 0.0004 & $7.90 \mathrm{E}-01$ \\
\hline
\end{tabular}

${ }^{\text {a }}$ Number of non-missing genotypes

${ }^{\mathrm{b}}$ Regression coefficient

${ }^{\mathrm{c}}$ Regression $r$-squared

${ }^{\mathrm{d}}$ Wald test $P$ value
Table 7 Anti-GAD IgM reactivity QTL analysis in patient parents

\begin{tabular}{lllll}
\hline Marker & NMISS $^{\mathrm{a}}$ & Beta $^{\mathrm{b}}$ & $r^{2 \mathrm{c}}$ & $P^{\mathrm{d}}$ \\
\hline rs2180790 & 139 & -0.038 & 0.028 & $4.88 \mathrm{E}-02$ \\
rs1956596 & 140 & -0.039 & 0.030 & $3.92 \mathrm{E}-02$ \\
rs1950942 & 129 & -0.057 & 0.062 & $4.17 \mathrm{E}-03$ \\
rs1808152 & 114 & 0.021 & 0.007 & $3.71 \mathrm{E}-01$ \\
\hline
\end{tabular}

${ }^{\mathrm{a}}$ Number of non-missing genotypes

${ }^{\mathrm{b}}$ Regression coefficient

${ }^{\mathrm{c}}$ Regression $r$-squared

${ }^{\mathrm{d}}$ Wald test $P$ value

from antigen-driven affinity maturation occurring during adaptive immune responses. It has been demonstrated that anti-islet IgM reactivity is precociously detected in T1D (Hoppu et al. 2004b). In particular, one study reported increased concentration of circulating serum IgM in recently diagnosed T1D patients accompanied by increased $\operatorname{IgM}$ and also IgG binding to fixed rat islet cells (Decraene et al. 1992). Another study showed that positivity for both GAD and IA-2 antibodies at birth was associated with a 7.5-fold increased risk for developing T1D in a cohort of Danish children (Eising et al. 2011). We found that the levels of IgM with anti-GAD reactivity were significantly higher in T1D patients when compared to their parents. Strikingly, analysis of the genetic control of IgM antiGAD reactivity in T1D patients and non-affected parents by the $I G H$ locus converged in rs1950942, which maps in the IGHM region and was also the most associated with T1D. These results support the hypothesis that $I G H$ variants contribute to the genetic control of natural IgM self-reactivity. We speculate that deviations of the natural IgM reactivity could fuel chronic pancreatic auto-reactivity that generates IgG AutoAbs accompanying progression to T1D. Consistent with this hypothesis, rs 1950942 in the $I G H M$ was associated with positivity to $\operatorname{IgG}$ AutoAbs and with $\mathrm{IgG}$ autoantibody multi-reactive repertoire in T1D patients.

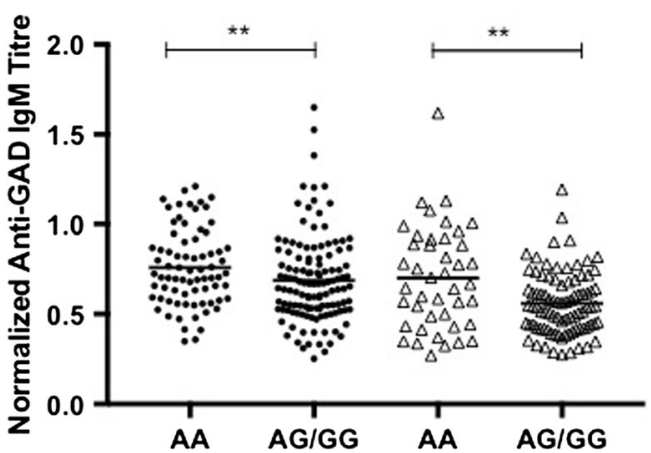

Fig. 4 T1D-susceptibility genotype at rs 1950942 is associated with increased levels of anti-GAD IgM. Normalized anti-GAD IgM titers in 200 patients (black circles) and 129 relatives (open triangles) stratified by the T1D-aasocited genotyped at $\operatorname{rs} 1950942$ ( $A A$ versus $A G / G G$ ). $* * P<0.01$, Mann-Whitney test (one-tailed) 
A GWAS remarked that the majority of T1D regions did not associate with autoantibody positivity (Plagnol et al. 2011). Also, HLA alleles associated with AutoAbs in T1D patients were shown to be distinct from T1D susceptibility conferred by the HLA locus (Howson et al. 2011). These results suggest that known T1D susceptibility loci were dissociated from the genetic determination of IgG AutoAbs in T1D patients.

Overall, our results suggest that the $I G H$ genetic variation is associated with T1D susceptibility by shaping IgM reactivity and subsequently predisposing to the generation of $\operatorname{IgG}$ AutoAbs in the course of the T1D natural history. Confirmation of our proposal that the $I G H$ locus takes part in the genetic component of T1D susceptibility warrants replication of these findings in larger patient collections.

Acknowledgements We are grateful to all participants in this study. We acknowledge the financial support of Fundação para a Ciência e a Tecnologia through the grant PPCDT/SAU-MMO/57955 and the fellowship SFRH/BPD/43631 to Nádia Duarte. We thank Dr. Íris Caramalho for the critical reading of the manuscript.

Authors' contributions IR generated the genotype and antibody data, analyzed the data, and wrote the manuscript. ND generated the antibody data, analyzed the data, and wrote the manuscript. GB generated the antibody data and analyzed the data. JC generated the genotype data. LGC, JB, RD, and JR contributed to the patient collection and clinical characterization. ZP generated the antibody data. MC contributed to the study design and wrote the manuscript. CPG contributed to the study design and coordination, analyzed the data, and wrote the manuscript. All authors approved final submission.

\section{Compliance with ethical standards}

Ethics approval and consent to participate All procedures performed in studies involving human participants were in accordance with the ethical standards of the institutional and/or national research committee and with the 1964 Helsinki declaration and its later amendments or comparable ethical standards. This investigation was conducted under the ethical permission obtained for the study entitled "Estudo da base genética da imunopatologia associada à Diabetes Tipo 1 na população portuguesa" granted by the Ethics Committee of the Associação Protectora dos Diabéticos de Portugal (APDP) in Lisbon. Written, informed consent was obtained from the adult participants or parents of each child participant.

Conflict of interest The authors declare that they have no conflict of interest.

Open Access This article is distributed under the terms of the Creative Commons Attribution 4.0 International License (http:// creativecommons.org/licenses/by/4.0/), which permits unrestricted use, distribution, and reproduction in any medium, provided you give appropriate credit to the original author(s) and the source, provide a link to the Creative Commons license, and indicate if changes were made.

\section{References}

Aktay AN, Lee PC, Kumar V et al (2001) The prevalence and clinical characteristics of celiac disease in juvenile diabetes in Wisconsin. $\mathrm{J}$ Pediatr Gastroenterol Nutr 33:462-465

Andersson A, Ekstrand-Hammarström B, Eriksson B et al (1994) Neonatal treatment with monoclonal natural antibodies restores a normal pattern of $\mathrm{VH}$ gene utilization in the non-obese diabetic mouse. Int Immunol 6:623-630

Bach JF (1994) Insulin-dependent diabetes mellitus as an autoimmune disease. Endocr Rev 15:516-542

Bao F, Yu L, Babu S et al (1999) One third of HLA DQ2 homozygous patients with type 1 diabetes express celiac disease-associated transglutaminase autoantibodies. J Autoimmun 13:143-148. doi: 10.1006/jaut.1999.0303

Barera G, Bonfanti R, Viscardi M et al (2002) Occurrence of celiac disease after onset of type 1 diabetes: a 6-year prospective longitudinal study. Pediatrics 109:833-838. doi:10.1542/peds.109.5.833

Barker JM, Yu J, Yu L et al (2005) Autoantibody "subspecificity" in type 1 diabetes: risk for organ-specific autoimmunity clusters in distinct groups. Diabetes Care 28:850-855

Burton PR, Clayton DG, Cardon LR et al (2007) $<<<<$ duplicate $>>>>$ Genome-wide association study of 14,000 cases of seven common diseases and 3,000 shared controls. Nature 447:661

Carlsson AK, Axelsson IE, Borulf SK et al (1999) Prevalence of IgAantiendomysium and IgA-antigliadin autoantibodies at diagnosis of insulin-dependent diabetes mellitus in Swedish children and adolescents. Pediatrics 103:1248-1252. doi:10.1542/peds.103.6.1248

Chan O, Shlomchik MJ (1998) A new role for B cells in systemic autoimmunity: B cells promote spontaneous $\mathrm{T}$ cell activation in MRLlpr/lpr mice. J Immunol 160:51-59

Chan OT, Hannum LG, Haberman AM et al (1999) A novel mouse with B cells but lacking serum antibody reveals an antibody-independent role for B cells in murine lupus. J Exp Med 189:1639-1648. doi:10. 1084/jem.189.10.1639

Côrte-Real J, Duarte N, Tavares L, Penha-Gonçalves C (2012) Innate stimulation of B1a cells enhances the autoreactive $\operatorname{IgM}$ repertoire in the NOD mouse: implications for type 1 diabetes. Diabetologia 55:1761-1772. doi:10.1007/s00125-012-2498-0

Coutinho A, Kazatchkine MD, Avrameas S (1995) Natural autoantibodies. Curr Opin Immunol 7:812-818

Dean BM, Becker F, McNally JM et al (1986) Insulin autoantibodies in the pre-diabetic period: correlation with islet cell antibodies and development of diabetes. Diabetologia 29:339-342

Decraene T, Vandewalle C, Pipeleers D et al (1992) Increased concentrations of total IgM at clinical onset of type 1 (insulin-dependent) diabetes: correlation with IgM binding to cells. Clin Chem 38: $1762-1767$

Eising S, Nilsson A, Carstensen B et al (2011) Danish children born with glutamic acid decarboxylase- 65 and islet antigen- 2 autoantibodies at birth had an increased risk to develop type 1 diabetes. Eur J Endocrinol 164:247-252. doi:10.1530/EJE-10-0792

Elliott C, Lindner M, Arthur A et al (2012) Functional identification of pathogenic autoantibody responses in patients with multiple sclerosis. Brain 135:1819-1833. doi:10.1093/brain/aws105

Harbers SO, Crocker A, Catalano G et al (2007) Antibody-enhanced cross-presentation of self antigen breaks $\mathrm{T}$ cell tolerance. J Clin Invest 117:1361-1369. doi:10.1172/JCI29470

Harris DP, Haynes L, Sayles PC et al (2000) Reciprocal regulation of polarized cytokine production by effector B and T cells. Nat Immunol 1:475-482. doi:10.1038/82717

Hawa MI, Fava D, Medici F et al (2000) Antibodies to IA-2 and GAD65 in type 1 and type 2 diabetes: isotype restriction and polyclonality. Diabetes Care 23:228-233. doi:10.1128/AAC.03728-14 
Hoppu S, Ronkainen MS, Kimpimäki T et al (2004a) Insulin autoantibody isotypes during the prediabetic process in young children with increased genetic risk of type 1 diabetes. Pediatr Res 55:236-242. doi:10.1203/01.PDR.0000100905.41131.3F

Hoppu S, Ronkainen MS, Kulmala P et al (2004b) GAD65 antibody isotypes and epitope recognition during the prediabetic process in siblings of children with type I diabetes. Clin Exp Immunol 136: 120-128. doi:10.1111/j.1365-2249.2004.02416.x

Howson JMM, Stevens H, Smyth DJ et al (2011) Evidence that HLA class I and II associations with type 1 diabetes, autoantibodies to GAD and autoantibodies to IA-2, are distinct. Diabetes 60:26352644. doi:10.2337/db11-0131

Kimpimäki T, Kulmala P, Savola K et al (2002) Natural history of betacell autoimmunity in young children with increased genetic susceptibility to type 1 diabetes recruited from the general population. J Clin Endocrinol Metab 87:4572-4579. doi:10.1210/jc.2002-020018

LaGasse JM, Brantley MS, Leech NJ et al (2002) Successful prospective prediction of type 1 diabetes in schoolchildren through multiple defined autoantibodies: an 8-year follow-up of the Washington state diabetes prediction study. Diabetes Care 25:505-511

Merbl Y, Zucker-Toledano M, Quintana FJ, Cohen IR (2007) Newborn humans manifest autoantibodies to defined self molecules detected by antigen microarray informatics. J Clin Invest 117:712-718. doi: 10.1172/JCI29943

Notkins AL, Åke L (2001) Autoimmune type 1 diabetes: resolved and unresolved issues. J Clin Invest 108:1247-1252

Perros P, McCrimmon RJ, Shaw G, Frier BM (1995) Frequency of thyroid dysfunction in diabetic patients: value of annual screening. Diabet Med 12:622-627

Pescovitz MD, Greenbaum CJ, Krause-Steinrauf H et al (2009) Rituximab, B-lymphocyte depletion, and preservation of beta-cell function. N Engl J Med 361:2143-2152. doi:10.1056/ NEJMoa0904452
Plagnol V, Howson JMM, Smyth DJ et al (2011) Genome-wide association analysis of autoantibody positivity in type 1 diabetes cases. PLoS Genet 7:e1002216. doi:10.1371/journal. pgen. 1002216

Purcell S, Neale B, Todd-Brown K et al (2007) PLINK: a tool set for whole-genome association and population-based linkage analyses. Am J Hum Genet 81:559-575. doi:10.1086/519795

Roep BO, Peakman M (2011) Diabetogenic T lymphocytes in human type 1 diabetes. Curr Opin Immunol 23:746-753

Silva DG, Daley SR, Hogan J et al (2011) Anti-islet autoantibodies trigger autoimmune diabetes in the presence of an increased frequency of islet-reactive CD4 T cells. Diabetes 60:2102-2111. doi:10.2337/ db10-1344

Umpierrez GE, Latif KA, Murphy MB et al (2003) Thyroid dysfunction in patients with type 1 diabetes: a longitudinal study. Diabetes Care 26:1181-1185. doi:10.2337/diacare.26.4.1181

Viale BA, Coutinho A, Freitas AA (1992) Differential expression of VH gene families in peripheral B cell repertoires of newborn or adult immunoglobulin H chain congenic mice. J Exp Med 175:14491456

Wong FS, Wen L, Tang M et al (2004) Investigation of the role of B-cells in type 1 diabetes in the NOD mouse. Diabetes 53:2581-2587. doi: 10.2337/diabetes.53.10.2581

Yu L, Robles DT, Abiru N et al (2000) Early expression of antiinsulin autoantibodies of humans and the NOD mouse: evidence for early determination of subsequent diabetes. Proc Natl Acad Sci U S A 97: 1701-1706. doi:10.1073/pnas.040556697

Ziegler AG, Hummel M, Schenker M, Bonifacio E (1999) Autoantibody appearance and risk for development of childhood diabetes in offspring of parents with type 1 diabetes: the 2-year analysis of the German BABYDIAB study. Diabetes 48:460-468. doi:10.2337/ diabetes.48.3.460 